\title{
The effects of oil displacement agents on the stability of water produced from ASP (alkaline/surfactant/polymer) flooding
}

\author{
Biao Wang ${ }^{a}$, Tao $\mathrm{Wu}^{\mathrm{b}}$, Yujiang $\mathrm{Li}^{\mathrm{a}}{ }^{*}$, Dejun Sun ${ }^{\mathrm{b}}$, Min Yang ${ }^{\mathrm{c}, * *}$, Yingxin Gao ${ }^{\mathrm{c}}$, Fengjuan $\mathrm{Lu}^{\mathrm{a}}, \mathrm{Xia}^{\mathrm{Li}}{ }^{\mathrm{a}}$

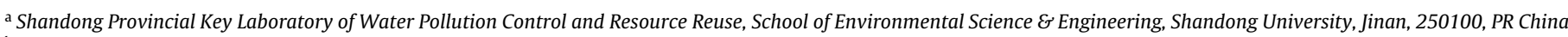 \\ ${ }^{\mathrm{b}}$ Key Laboratory of Colloid E' Interface Science of Education Ministry, Shandong University, Jinan, 250100, PR China \\ ' State Key Laboratory of Environmental Aquatic Chemistry, Research Center for Eco-Environmental Sciences, Chinese Academy of Sciences, Beijing, 100085, PR China
}

\section{A R T I C L E I N F O}

\section{Article history:}

Received 28 June 2010

Received in revised form 23 October 2010

Accepted 26 November 2010

Available online 10 December 2010

\section{Keywords:}

Simulated produced water

ASP flooding technology

Interfacial property

Oil-water separation

Leaching solution of alkaline white mud

\begin{abstract}
A B S T R A C T
Alkaline/surfactant/polymer (ASP) flooding technology has been successfully used in Chinese oil fields, such as Daqing and Shengli. However, water produced from ASP flooding contains large quantities of residual chemicals (alkali, surfactant and polymer) making it a complex and stable emulsion system which is difficult to treat. The emulsion stability of water produced from ASP flooding was investigated by conducting settling experiments and measuring the oil-water interfacial properties. The experimental results showed that the addition of polymer (HPAM, hydrolyzed polyacrylamide) degrades the emulsion stability when its concentration is below $300 \mathrm{mg} / \mathrm{L}$ for the $1.2 \times 10^{7} \mathrm{MW}$ polymer, and $800 \mathrm{mg} / \mathrm{L}$ for the $3.0 \times 10^{6} \mathrm{MW}$ polymer. But it enhances the emulsion stability when polymer concentrations are above those levels. At low polymer concentrations, flocculation induced by the polymer on oil droplets in the produced water is the dominant factor, while at high polymer concentrations the produced water viscosity plays an important role in the emulsion stability. The adsorption of surfactant on the oil-water interface increases the zeta potentials and decreases interfacial tension, and thus remarkably enhances the emulsion stability. Furthermore, the emulsion stability is enhanced gradually with the increase of $\mathrm{NaOH}$ concentration up to $300 \mathrm{mg} / \mathrm{L}$ due to the increase of zeta potentials and decrease of interfacial tension, and then weakened with the further increase of $\mathrm{NaOH}$ concentration, which is attributed to the decreased strength of the interfacial film. A pilot experiment for the treatment of simulated water was done, and the result showed that the simulated produced water from ASP can be successfully treated by using a leaching solution of alkaline white mud.
\end{abstract}

(C) 2010 Elsevier B.V. All rights reserved.

\section{Introduction}

In crude oil extraction, aqueous solutions containing large quantities of chemicals can be injected into the strata to drive the crude oil out of the ground. This process is often termed chemical flooding or tertiary oil extraction. In recent years, technologies for tertiary oil extraction have been relatively well developed. Alkaline-surfactant-polymer (ASP) flooding technology (injecting an aqueous solution containing alkali, surfactant and polymer) is an important technology in tertiary oil extraction, which has been found to enhance oil recovery by over $20 \%$ [1]. Therefore, this technology has been used in several different oil fields in China, such as Daqing and Shengli [2], to enhance oil recovery. However, the oil fields then face with new problems. Produced water is gener-

\footnotetext{
* Corresponding author. Tel.: +86 531 88363358; fax: +86 53188363358 .

** Corresponding author. Tel.: +86 10 62923475; fax: +86 1062923475.

E-mail addresses: yujiang@sdu.edu.cn (Y. Li), yangmin@mail.rcees.ac.cn (M. Yang).
}

ated after dehydration of the produced liquid (a mixture of oil and water from the oil wells). And due to the use of alkali, surfactant and polymer in the injected aqueous solution in ASP flooding technology, the produced water from this process contains large numbers of residual chemicals. Thus the water produced from ASP flooding forms a complex and stable emulsion system [3] and it is more difficult to treat than that from water flooding.

In a series of articles, Deng and co-workers have investigated the properties of oil-in-water emulsions based on the Daqing crude oil [1,4-6]. Their interest has been focused on the stability of oil droplets in the produced water and treatment of the produced water using a novel crossflow oil-water separator. Furthermore, flocculation and demulsification have been conducted to remove the stable oil droplets in the produced water and Deng et al. found that demulsification was an effective method to accelerate oil-water separation for the produced water. All these studies have given us insight into the problems relating to emulsion stability and treatment of produced water from ASP flooding. However, the stabilization mechanism of produced water from ASP flooding has not been fully studied and to our knowledge, no effective technologies 
can meet the need for the treatment of produced water from ASP flooding system in oilfields.

It is now generally recognized that the zeta potentials, interfacial tension and interfacial rheology have effects on the stability of emulsions [7]. Thus, in this study, simulated produced water from ASP flooding was prepared and the effects of oil displacement agents (alkali, surfactant and polymer) on the emulsion stability was investigated in terms of oil-water separation of produced water, interfacial tension, interfacial rheology, zeta potential, bulk phase viscosity and sizes of oil droplets. Furthermore, we investigated treatment of the simulated produced water from ASP flooding using a leaching solution of alkaline white mud.

\section{Materials and methods}

\subsection{Materials}

The crude oil used in this study was obtained from the Shengli oilfield in China, with a water content of less than $0.5 \%$, and a density of $865 \mathrm{~kg} / \mathrm{m}^{3}$ and viscosity $50.38 \mathrm{mPa}$, at $45^{\circ} \mathrm{C}$. The partly hydrolyzed polyacrylamide (HPAM) used as polymer was purchased from East Asiatic Company (Japan), with average molecular weights (MW) of $3.0 \times 10^{6}$ and $1.2 \times 10^{7}$, and a degree of hydrolysis of about $20-30 \%$. The surfactant (petroleum sulphonate, WPS) was supplied by Shengli Engineering \& Consulting Co., Ltd. The alkali used in this study was $\mathrm{NaOH}$ and of reagent grade. Other materials were all analytical reagents.

\subsection{Preparation of simulated produced water}

According to the underground water and discharge water qualities in the Shengli oilfield, mineral water was prepared firstly, with salt concentrations as follows (mg/L), $\mathrm{NaCl} 1600, \mathrm{NaHCO}_{3} 2600$, $\mathrm{Na}_{2} \mathrm{CO}_{3} 300, \mathrm{Na}_{2} \mathrm{SO}_{4} 40, \mathrm{CaCl}_{2} 40, \mathrm{MgCl}_{2}$ 40. Then the mineral water was used to prepare simulated water produced from ASP flooding. The preparation process was as follows: $210 \mathrm{~g}$ of mineral water with $0.1 \%$ WPS and $90 \mathrm{~g}$ of crude oil were added to a $500 \mathrm{ml}$ beaker and the mixture was heated to $45^{\circ} \mathrm{C}$ in a water bath for $1 \mathrm{~h}$. Then the mixture was emulsified for $5 \mathrm{~min}$ at $15,000 \mathrm{rpm}$ with an emulsifier to obtain a $30 \%$ oil-in-water emulsion. Next, $0.5 \mathrm{~g}$ of $30 \%$ oil-in-water emulsion was added to $99.5 \mathrm{ml}$ of mineral water with different concentrations of oil displacement agents, and the mineral water was shaken to produce simulated produced water with an oil concentration of $1500 \mathrm{mg} / \mathrm{L}$.

\subsection{Oil concentration determination}

To determine the concentration of oil in the water samples, $100 \mathrm{ml}$ of the prepared produced water in a $100-\mathrm{ml}$ beaker was allowed to settle for $4 \mathrm{~h}$ at $45^{\circ} \mathrm{C}$ in an air bath. Then $30 \mathrm{ml}$ of the water was taken from the bottom of the beaker using a syringe and injected into a separating funnel. The $\mathrm{pH}$ of the water was adjusted to about 2.0 using $1: 1(\mathrm{v} / \mathrm{v}) \mathrm{HCl}$, and then extracted two times by petroleum ether. The absorbance of the extract was determined by a TU- 1810 type UV-vis spectrophotometer at $430 \mathrm{~nm}$ $[8,9]$. Petroleum ether served as a reference. The oil contents in the water were calculated according to the standard curve. Oil concentrations represent the oil-water separation properties of the simulated produced water, namely the emulsion stability of the produced water.

\subsection{Viscosity measurement}

The viscosity of the produced water was determined by NDJ9S digital viscometer from Shanghai Balance Company (China). In the measurement, the rotor was a number one rotor, the shear rate was $60 \mathrm{rpm}$ and the temperature of the produced water was kept at $45^{\circ} \mathrm{C}$ constantly.

\subsection{Oil-water interfacial tension and interfacial dilatational rheology measurement}

Interfacial dilatational modulus $(\varepsilon)$ is defined as follows:

$\varepsilon=\frac{d \gamma}{d \ln A}$,

where $\gamma$ is interfacial tension and $A$ is the area of the interface. Interfacial dilatational modulus has both a real and imaginary component defined for an oscillating area as follows [10,11]:

$\varepsilon=\varepsilon_{d}+i \omega \eta_{d}$,

where $\varepsilon_{d}$ is the dilational elasticity, $\omega$ is the frequency of the oscillations, and $\eta_{d}$ is the dilational viscosity.

An interfacial rheometer (I.T. Concept, France) was used to measure the interfacial tension and interfacial dilatational modulus. The crude oil was loaded into a syringe and then injected through a Ushaped needle into an optical glass cuvette containing simulated produced water from ASP flooding with different concentrations of oil displacement agents. The profile of the droplet was captured using a CCD camera and analyzed using a video image profile digitizer board connected to a personal computer. When the experiments were carried out, the volume of the drop was manipulated by software so that the area of the droplet oscillated sinusoidally. The interfacial tension and droplet surface area were both measured and the interfacial dilatational modulus was calculated using Fourier analysis. The cuvette was placed in a jacket controlled with a water bath at $45^{\circ} \mathrm{C}$.

\subsection{Determination of zeta potential of oil droplets}

The zeta potential of oil droplets was measured by a Zeta plus Zeta apparatus (Shanghai, Zhongchen Company). First, $100 \mathrm{ml}$ of the produced water was allowed to settle for $4 \mathrm{~h}$ at $45^{\circ} \mathrm{C}$ in an air bath, and then $5 \mathrm{ml}$ of water was taken from the beaker for zeta potential measurement.

\subsection{Droplet size distribution analysis}

The droplet size distribution for the produced water was measured by a laser diffraction method using a Mastersizer 2000 (Malvern Instruments Ltd., Worcestershire, UK). First, $500 \mathrm{ml}$ of distilled water was added to a beaker, and heated up to $45^{\circ} \mathrm{C}$ in a water bath. A proper volume of produced water containing oil droplets was then diluted to a droplet concentration of less than about $0.05 \mathrm{wt} \%$ (to eliminate multiple scattering effects), and gently stirred (to increase the homogeneity) prior to measurement. The initial size distribution was measured immediately after the produced water was prepared and the size distribution was measured again approximately $2 \mathrm{~h}$ after the preparation.

\subsection{A leaching solution of alkaline white mud}

Alkaline white mud, collected from the Shandong Aluminum Plant, was dried at $105^{\circ} \mathrm{C}$ overnight, disaggregated, and ground to pass through a 200 mesh sieve for use. Chemical composition of the white mud was determined by X-ray fluorescence (ZSX Primus II, Rigaku Corporation) and the main metallic elements are calcium, magnesium, iron and aluminum. And we prepared a treatment agent (leaching solution of alkaline white mud) by the reaction of alkaline white mud and $1.5 \mathrm{~mol} / \mathrm{L}$ dilute $\mathrm{HCl}$ solution and measured the solid content of the leaching solution $(0.1027 \mathrm{~g} / \mathrm{ml}$, dosage of leaching solution is based on the solid content). 

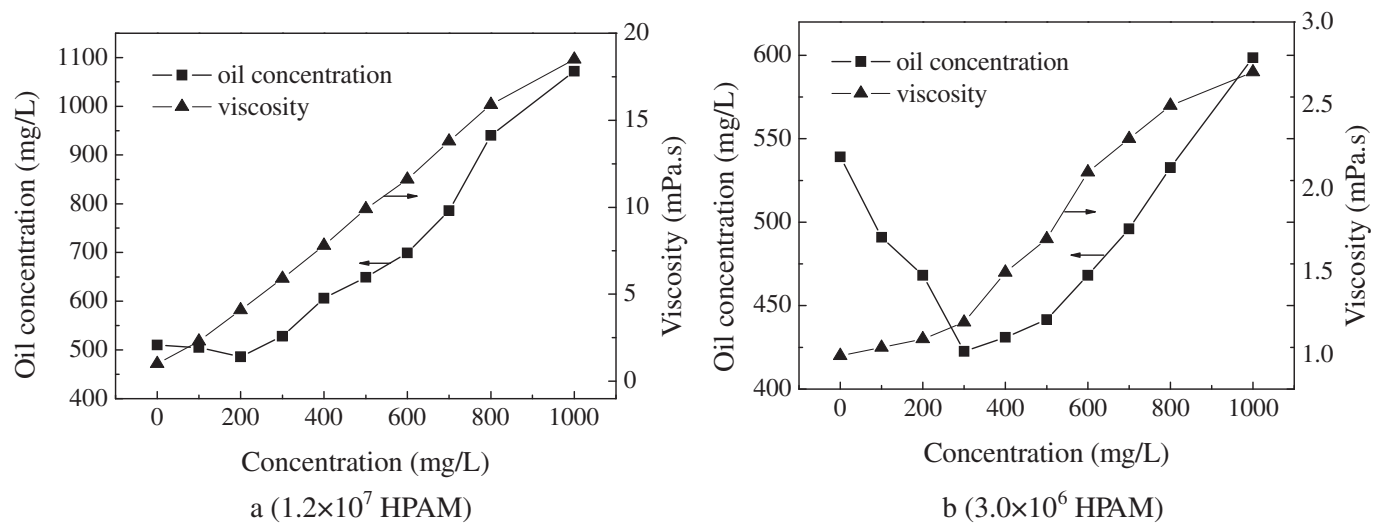

Fig. 1. Effects of HPAM on oil-water separation and viscosity of produced water.

\subsection{Beaker experiment for the pilot study on the treatment of simulated water}

Experiments were conducted using a batch method by adding the leaching solution of white mud into $500-\mathrm{ml}$ beakers containing $400 \mathrm{ml}$ simulated water of desired concentrations (oil, alkaline surfactant and polymer) and $\mathrm{pH} 9.5$, After addition of $10-20 \mathrm{ml}$ leaching solution of white mud, the simulated water was stirred rapidly at $160 \mathrm{rpm}$ for $1 \mathrm{~min}$, followed by a slow stir phase at $40 \mathrm{rpm}$ for $5 \mathrm{~min}$, and then a $5 \mathrm{~min}$ settling time. The supernatant sample was extracted by a syringe from about $2 \mathrm{~cm}$ below the water surface for analysis of oil contents and COD.

\section{Results and discussion}

\subsection{Effects of oil displacement agents on emulsion stability of simulated produced water}

The emulsion stability of simulated produced water was assessed by measuring the oil concentrations after settling experiments as a function of concentrations. The oil concentration is an indicator of the emulsion stability of the simulated produced water.

\subsubsection{Effects of HPAM concentration}

The effects of HPAM concentrations on emulsion stability and viscosity of produced water were studied with the concentrations of $\mathrm{NaOH}$ and WPS at $800 \mathrm{mg} / \mathrm{L}$ and $600 \mathrm{mg} / \mathrm{L}$, respectively, as shown in Fig. 1. HPAM is a high molecular weight polymer and can increase the viscosity of the produced water. The effect of low molecular weight $\left(\right.$ LMW) HPAM $\left(3.0 \times 10^{6}\right)$ on the viscosity is lower than that of high molecular weight (HMW) HPAM $\left(1.2 \times 10^{7}\right)$. It also can be observed that the oil concentrations of the produced water fall at first, and then go up as the HPAM concentrations increase. For HMW HPAM, Fig. 1(a) indicates that the oil concentration decreased from $510 \mathrm{mg} / \mathrm{L}$ to $486 \mathrm{mg} / \mathrm{L}$ when the HPAM concentration increased from 0 to $200 \mathrm{mg} / \mathrm{L}$. The oil concentration then increased from $486 \mathrm{mg} / \mathrm{L}$ to $1072 \mathrm{mg} / \mathrm{L}$ when the HPAM concentration increased further from 200 to $1000 \mathrm{mg} / \mathrm{L}$. For LMW HPAM, as shown in Fig. 1(b), the profile of oil concentrations exhibits a $\mathrm{V}$-shape. When the HPAM concentration was $300 \mathrm{mg} / \mathrm{L}$, the oil concentration reached a minimum value of $422 \mathrm{mg} / \mathrm{L}$. When the HPAM concentration is less than $300 \mathrm{mg} / \mathrm{L}$ and $800 \mathrm{mg} / \mathrm{L}$ for HMW and LMW HPAM, respectively, the corresponding oil concentrations of produced water are less than that of $0 \mathrm{mg} / \mathrm{L}$ HPAM. These differences for HMW and LMW HPAM may be due to the difference of the produced water's viscosities. For example, when the concentration of HMW HPAM is $500 \mathrm{mg} / \mathrm{L}$, the viscosity is $9.9 \mathrm{mPa} \mathrm{s}$, but the

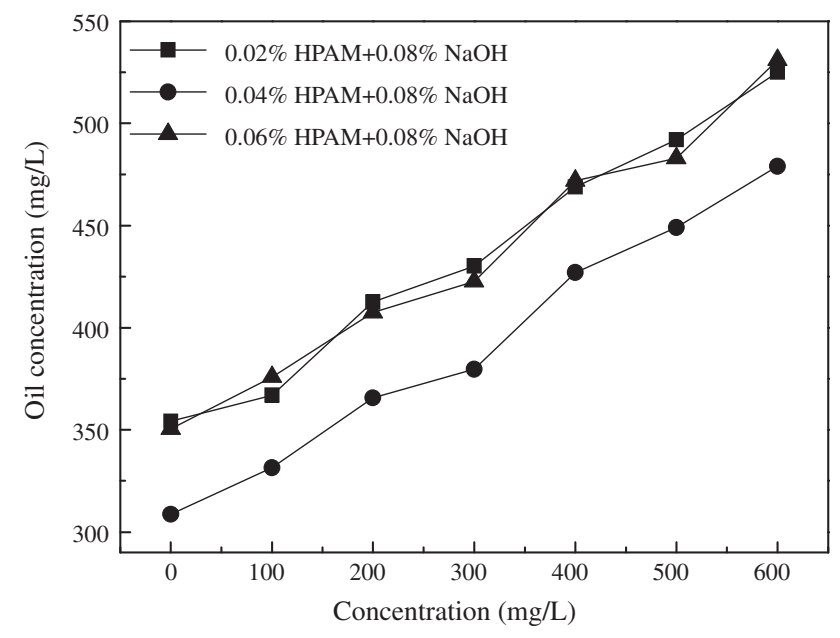

Fig. 2. Effects of surfactant on oil-water separation in the produced water.

viscosity is only $1.7 \mathrm{mPa}$ s when the concentration of LMW HPAM is $500 \mathrm{mg} / \mathrm{L}$. According to the Stokes equation [12], the rising velocity of an oil droplet is in reverse proportion to the viscosity, therefore high viscosity can prevent oil-water separation. The results illustrate that HPAM degrades the emulsion stability when its concentration is low, but enhances the emulsion stability when its concentration is above $300 \mathrm{mg} / \mathrm{L}$ and $800 \mathrm{mg} / \mathrm{L}$ for HMW and LMW HPAM, respectively.

\subsubsection{Effects of surfactant concentration}

The effects of WPS on oil concentrations were investigated with the concentration of $\mathrm{NaOH}$ at $800 \mathrm{mg} / \mathrm{L}$ and HPAM (the following experiments only use LMW HPAM as material) at 200, 400 and $600 \mathrm{mg} / \mathrm{L}$ (Fig. 2). Oil concentrations of produced water increased with the increase of WPS concentration, namely the emulsion stability of the produced water is enhanced with the addition of WPS. When the concentrations of HPAM are 200,400 and $600 \mathrm{mg} / \mathrm{L}$, the effects of WPS on oil concentration are nearly the same. And when the concentration of WPS increases from 0 to $600 \mathrm{mg} / \mathrm{L}$, the oil concentrations all increase by $160 \mathrm{mg} / \mathrm{L}$ for the three HPAM concentrations. The WPS surfactant can adsorb to the surface of the oil droplets, and its polar head groups extend into the water phase and the non-polar tails extend into the oil phase, so the oil-water interfacial properties may be changed [13-15]. The adsorption of WPS can enhance the emulsion stability of the produced water significantly. 


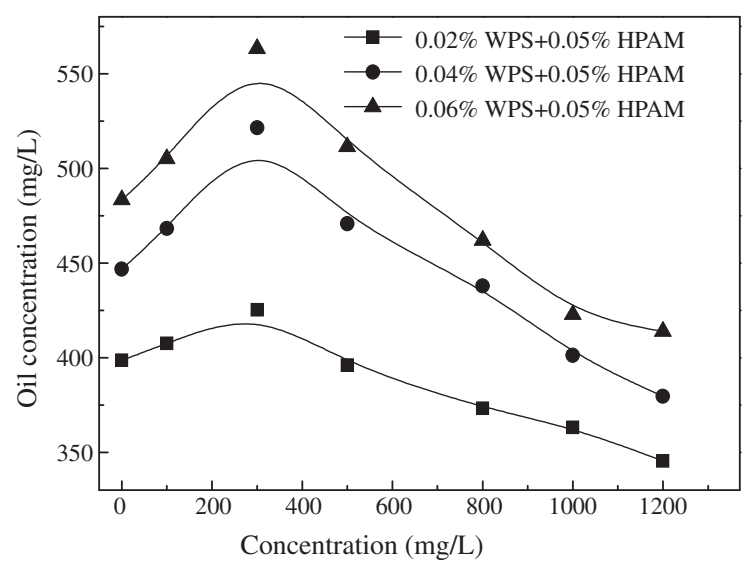

Fig. 3. Effects of $\mathrm{NaOH}$ on oil-water separation in the produced water.

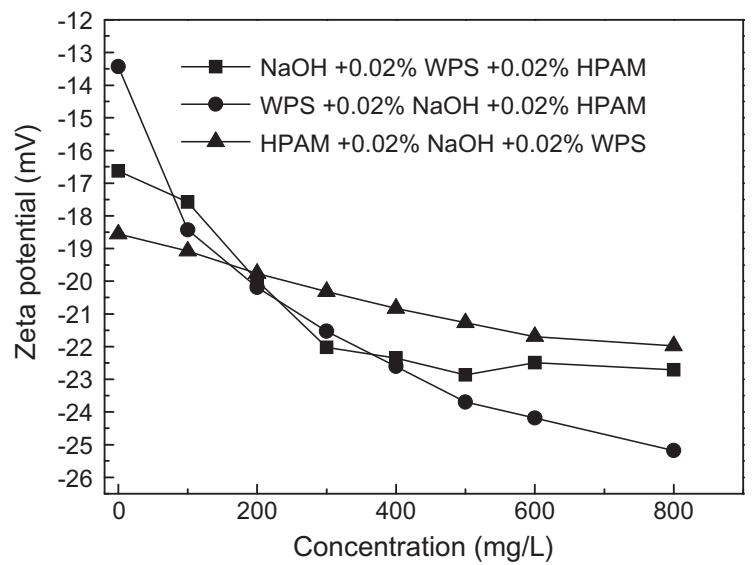

Fig. 4. Effects of oil displacement agents on zeta potential.

\subsubsection{Effects of $\mathrm{NaOH}$ concentration}

Fig. 3 illustrates the effects of $\mathrm{NaOH}$ on oil concentrations of the produced water. The oil-water separation experiments were carried out with the concentrations of HPAM at $500 \mathrm{mg} / \mathrm{L}$, and WPS at 200,400 and $600 \mathrm{mg} / \mathrm{L}$. It can be seen that the oil concentrations of the produced water increase initially when the $\mathrm{NaOH}$ concentration is increased from 0 to $300 \mathrm{mg} / \mathrm{L}$. The oil concentrations then decrease with the further increase of $\mathrm{NaOH}$ concentration. When the concentration of WPS is $200 \mathrm{mg} / \mathrm{L}$, the effect of $\mathrm{NaOH}$ on oil concentrations is somewhat different compared with the samples with $400 \mathrm{mg} / \mathrm{L}$ and $600 \mathrm{mg} / \mathrm{L}$ WPS. When the concentrations of $\mathrm{NaOH}$

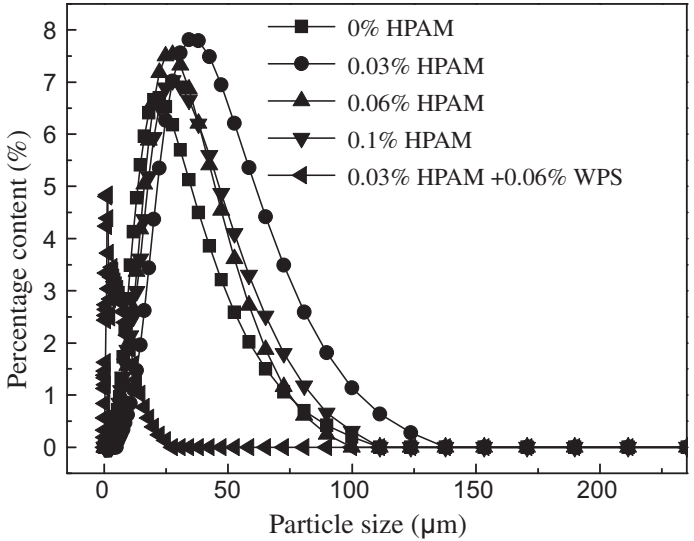

Fig. 6. Effects of HPAM and surfactant on sizes of oil droplets. Size distributions were measured after oscillation.

increased from $0 \mathrm{mg} / \mathrm{L}$ to $300 \mathrm{mg} / \mathrm{L}$, the increase of oil concentration is $26.6 \mathrm{mg} / \mathrm{L}, 74.7 \mathrm{mg} / \mathrm{L}$ and $79.8 \mathrm{mg} / \mathrm{L}$ for $200 \mathrm{mg} / \mathrm{L}, 400 \mathrm{mg} / \mathrm{L}$ and $600 \mathrm{mg} / \mathrm{L}$ WPS, respectively. The addition of $\mathrm{NaOH}$ can enhance the emulsion stability when its concentration is below $300 \mathrm{mg} / \mathrm{L}$, but it decreases the emulsion stability when the $\mathrm{NaOH}$ concentration is above $300 \mathrm{mg} / \mathrm{L}$.

\subsection{Effects of oil displacement agents on oil-water interfacial properties}

The emulsion stability of the produced water was determined by measuring the oil-water interfacial properties, such as interfacial tension (IFT) [16], strength of interfacial film [17-19], zeta potential $[20,21]$ and steric stabilization $[22,23]$. And these parameters were affected by the residual oil displacement agents in the produced water. Therefore, we investigated the effects of the oil displacement agents on the interfacial properties to find the mechanisms of stability of the emulsions in the produced water from ASP flooding.

HPAM is a water soluble polymer, and HPAM molecules can diffuse to the oil-water interface from the aqueous phase, but the adsorption of HPAM is quite weak due to the slight interfacial activity of HPAM. Thus, HPAM concentrations effect on the oil-water interfacial properties insignificant, as shown in Figs. 4 and 5. The zeta potential increases slowly with the increase of HPAM concentration, and HPAM hardly has any effect on IFT and viscoelastic modulus $(E)$. It can be seen from Fig. 6 that HPAM can promote the coalescence of oil droplets. The peak in the size distribution increased and shifted to larger sizes with the addition of HPAM. In addition, the tail of the drop size distribution became larger with the addition of HPAM. However, the floccu-
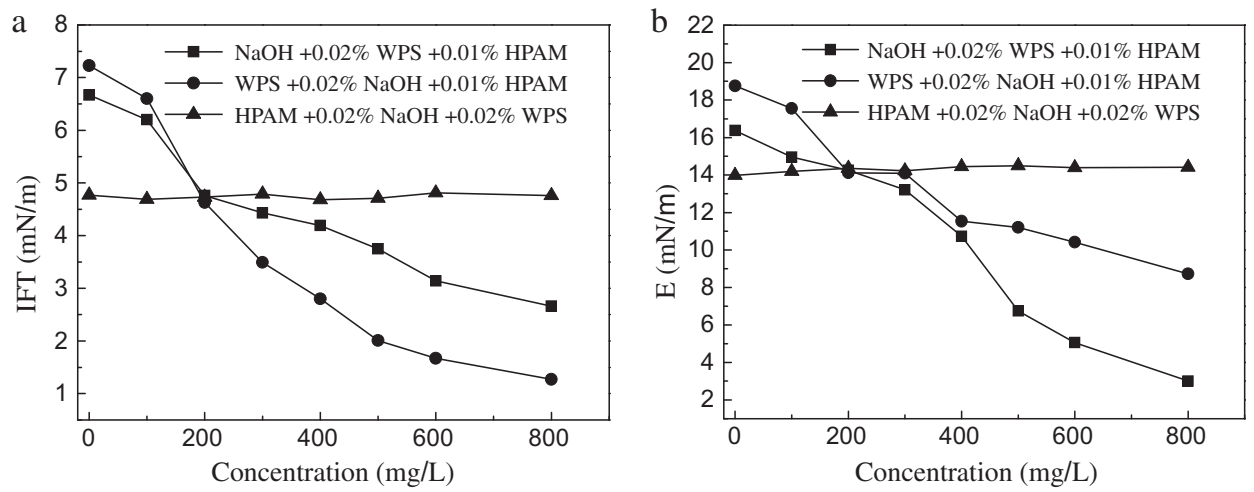

Fig. 5. Effects of oil displacement agents on interfacial tension and viscoelastic modulus. 
lation of HPAM decreased at higher HPAM concentrations, such as 600 and $1000 \mathrm{mg} / \mathrm{L}$ HPAM compared with $300 \mathrm{mg} / \mathrm{L}$. When HPAM concentration is below $300 \mathrm{mg} / \mathrm{L}$, the viscosity and zeta potential increase slightly, but the size of the oil droplets augments remarkably, therefore oil-water separation is accelerated and the emulsion stability is degraded with the HPAM concentration [16]. Yet at HPAM concentrations above $300 \mathrm{mg} / \mathrm{L}$, the effects of viscosity increase gradually (Fig. 1(b)). The drainage velocity of the liquid film between adjoining oil droplets decreases due to the viscosity increase. Thus, the flocculation of HPAM decreases and the emulsion stability increases with the increasing HPAM concentration. Overall, the viscosity change of produced water due to HPAM is the main factor for the emulsion stability, while the changes in zeta potential, IFT and $E$ are relatively insignificant. It may be related to the polymer depletion effect [24].

WPS is an anionic petroleum sulphonate surfactant and can be adsorbed to the surface of oil droplets with its non-polar tail attaching to oil phase and polar head group extending into the water phase, therefore the zeta potential increases significantly with the WPS content, as shown in Fig. 4. In addition, the interfacial properties have a great change due to the high interfacial activity of WPS. Fig. 5 shows that the IFT and elastic modulus, $E$, decrease with the WPS concentration. It was found that some interface active agents in crude oil, such as asphaltene, take part in the formation of the interfacial films on the crude oil-water interface, and the interfacial films possess a framework and a certain strength $[25,26]$. But with the addition of WPS, parts of the active agents are replaced by WPS, thus the original structure of interfacial film and the interaction force of interface molecules are changed, and IFT and $E$ decrease. Though the addition of WPS decreases the strength of the oil-water interfacial films, lower IFT enhances the inherent stability of oil droplets and higher zeta potential increases the electrostatic repulsive force between the droplets, therefore the addition of WPS prevents their coalescence. The double electric layer effect is likely the most significant factor.

As shown in Fig. 6, the peak in the distribution decreased and shifted to smaller size when the produced water contained WPS. Thus, the emulsion stability of the produced water increases with the increase of WPS concentration.

The effect of $\mathrm{NaOH}$ on zeta potential is shown in Fig. 4. Zeta potential increases with $\mathrm{NaOH}$ concentration, but the increase is slight after $\mathrm{NaOH}$ concentration is above $300 \mathrm{mg} / \mathrm{L}$. NaOH can react with acidic components of crude oil and form soap-like interfacial active components [27], that can be adsorbed to the oil-water interfacial film like the surfactant, so the zeta potential increases. But the quantity of acidic components is finite, therefore the increase of zeta potential is not obvious when $\mathrm{NaOH}$ concentration exceeds $300 \mathrm{mg} / \mathrm{L}$. Also, the adsorption of soap can affect IFT and E, and

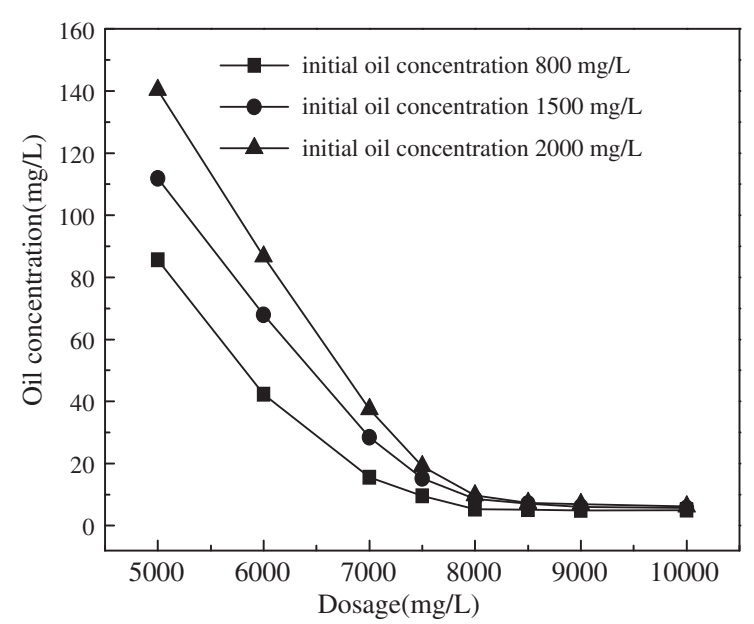

Fig. 7. Effects of dosage of leaching solution of alkaline white mud on the treatment effect.

the loss of interfacial active agents in the interfacial film by reacting with $\mathrm{NaOH}$, so the change of interface structure is significant. As shown in Fig. 5, IFT and $E$ both decrease with the increase of $\mathrm{NaOH}$. At low $\mathrm{NaOH}$ concentrations (below $300 \mathrm{mg} / \mathrm{L}$ ), the zeta potential increases gradually, the strength of interfacial film is comparatively high, and the low IFT enhance the stability of the oil droplet itself, thus the emulsion stability of the produced water increases with the $\mathrm{NaOH}$ content. At high $\mathrm{NaOH}$ concentrations (above $300 \mathrm{mg} / \mathrm{L}$ ), the zeta potential is almost the same as that at low $\mathrm{NaOH}$ concentrations, but the strength of the interfacial film is greatly decreased, therefore the emulsion stability is decreased by degrees.

\subsection{The pilot study for the treatment of simulated water}

Given the high emulsion stability of water produced from ASP flooding, some conventional technologies have been found to be ineffective in the treatment of this water. There is a strong interaction between surfactant, polymer and hydroxides, hydroxy complex, which has been widely used in mineral floatation $[28,29]$. Therefore, the hydroxides and hydroxy complex produced from aqueous phase may have effect on the treatment of water produced from ASP flooding which contain some surfactant and HPAM. Then, we treated the simulated water produced by ASP flooding using a leaching solution of alkaline white mud at concentrations of alkali, surfactant and polymer of 2800,500 and $600 \mathrm{mg} / \mathrm{L}$, respectively. The experimental results (Fig. 7) showed that it is effective to treat the ASP flooding produced water by using a leaching solution of
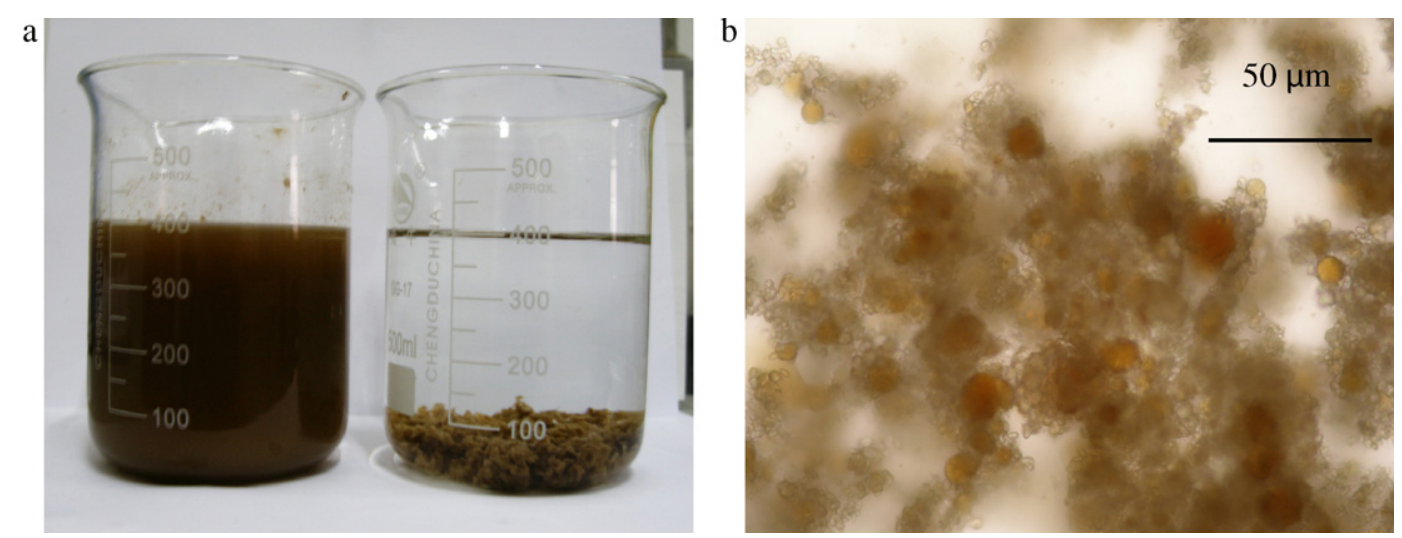

Fig. 8. The photograph of treatment effect (a) and the optical microscope image of precipitate (b). 
alkaline white mud. When the dosage is above $8000 \mathrm{mg} / \mathrm{L}$, the oil concentrations after treatment are less than $10 \mathrm{mg} / \mathrm{L}$ which meets the demand of oilfield refill water and the COD is less than $150 \mathrm{mg} / \mathrm{L}$. A photograph of treatment effect and an optical microscope image of the precipitate are shown in Fig. 8. We deduce that the leaching solution of alkaline white mud reacts with alkali and the other substances in the produced water including oil, WPS and HPAM, forming a co-precipitate with the reaction products. Therefore, the water and oil in the ASP flooding water are separated.

\section{Conclusions}

The wastewater produced from ASP flooding is a complex emulsion system with high emulsion stability, and it is difficult to treat. The effects of oil displacement agents on emulsion stability of water produced from ASP flooding are various. HPAM increased viscosity, promoted the coalescence of oil droplets, and affected the IFT and $E$ slightly. With the increase of HPAM concentration, the emulsion stability of produced water initially decreased and then increased. At low HPAM concentration, the flocculation is the most important effect, while at high HPAM concentration; destabilization is inhibited due to the high viscosity. WPS decreases the IFT and $E$, and increases the zeta potentials greatly. The emulsion stability is enhanced gradually with the increase of surfactant concentration, and the double electric layer effect is the most significant factor. $\mathrm{NaOH}$ increases the zeta potential, and decreases IFT and $E$. When $\mathrm{NaOH}$ concentration is low, the double electric layer effect dominates, but when $\mathrm{NaOH}$ concentration is high, the strength of the interfacial film plays the leading role.

The leaching solution of alkaline white mud can be used for treatment of the wastewater from ASP flooding. Consequently, we are investigating the treatment mechanism of the leaching solution.

\section{Acknowledgements}

This work was supported by the National Hi-Tech Research and Development Program of China (2009AA063901), the Innovation Program of the Universities in Jinan (201004043) and China National Petroleum Corporation innovation foundation (2009D5006-02-03). The authors thank Dr. Pamela Holt for revising the English in the manuscript.

\section{References}

[1] S. Deng, G. Yu, Z. Jiang, R. Zhang, Y. Ting, Destabilization of oil droplets in produced water from ASP flooding, Colloids Surf. A: Physicochem. Eng. Aspects 252 (2005) 113-119.

[2] J. Guo, Q. Liu, M. Li, Z. Wu, A.A. Christy, The effect of alkali on crude oil/water interfacial properties and the stability of crude oil emulsions, Colloids Surf. A: Physicochem. Eng. Aspects 273 (2006) 213-218.

[3] D. Wu, X. Meng, R. Zhang, H. Liang, F. Sun, Y. Li, N. Jiang, H. Lu, Stability and chemical treatment of Daqing oilfield ASP flooding produced water, Fine Chem. 18 (2001) 86-88

[4] R. Zhang, C. Liang, D. Wu, S. Deng, Characterization and demulsification of produced liquid from weak base ASP flooding, Colloids Surf. A: Physicochem. Eng. Aspects 290 (2006) 164-171.
[5] S. Deng, R. Bai, C.J. Paul, G. Yu, Z. Jiang, F. Zhou, Effects of alkaline/surfactant/polymer on stability of oil droplets in produced water from ASP flooding, Colloids Surf. A: Physicochem. Eng. Aspects 211 (2002) 275-284.

[6] S. Deng, R. Bai, C.J. Paul, Z. Jiang, G. Yu, F. Zhou, Z. Chen, Produced water from polymer flooding process in crude oil extraction: characterization and treatment by a novel crossflow oil-water separator, Sep. Purif. Technol. 29 (2002) 207-216.

[7] N. Panchev, K. Khristov, J. Czarnecki, D. Exerowa, S. Bhattacharjee, J. Masliyah, A new method for water-in-oil emulsion film studies, Colloids Surf. A: Physicochem. Eng. Aspects 315 (2008) 74-78.

[8] J. Yong, Z. Ping, T. Dong, J. Dong, Determination methods for oil concentration in the oily sludg, Environ. Sci. Manage. 33 (2008) 115-117.

[9] S. Deng, F. Zhou, Z. Chen, F. Xia, G. Yu, Z. Jiang, Effects of polyacrylamide on settling and separation of oil droplets in polymer flooding produced water Environ. Sci. 23 (2002) 69-71.

[10] F. Tomas, K.H. Finn, A. Kotzev, L. Andre, Adsorption and surface elastic properties of corresponding fluorinated and nonfluorinated cationic polymer films measured by drop shape analysis, Langmuir 17 (2001) 5256-5264.

[11] X. Cao, Y. Li, S. Jiang, H. Sun, C. Alain, L. Dou, A study of dilational rheological properties of polymers at interfaces, J. Colloid Interface Sci. 270 (2004) 295-298.

[12] S. Michael, E. Robert, R.S. Brian, A creaming study of weakly flocculated and depletion flocculated oil-in-water emulsions, Colloids Surf. A: Physicochem. Eng. Aspects 178 (2001) 265-276.

[13] H. Chen, L. Han, P. Luo, Z. Ye, The interfacial tension between oil and gemin surfactant solution, Surf. Sci. 552 (2004) L53-L57.

[14] D. Li, S. Lu, Y. Liu, D. Wang, The effect of biosurfactant on the interfacial tension and adsorption loss of surfactant in ASP flooding, Colloids Surf. A: Physicochem. Eng. Aspects 244 (2004) 53-60.

[15] L. Zhang, H. Xiao, H. Zhang, L. Xu, D. Zhang, Optimal design of a novel oil-water separator for raw oil produced from ASP flooding, J. Petrol. Sci. Eng. 59 (2007) 213-218.

[16] C.M. Chen, C.H. Lu, C.H. Chang, Y.M. Yang, J.R. Maa, Influence of pH on the stability of oil-in-water emulsions stabilized by a splittable surfactant, Colloids Surf. A: Physicochem. Eng. Aspects 170 (2000) 173-179.

[17] D. Li, Coalescence between two small bubbles or drops, J. Colloid Interface Sci. 163 (1994) 108-119.

[18] L. Xia, M. Li, P. Gu, G. Cao, S. Lu, Q. Liu, A study of shear viscosity of interfacia films containing alkyl metal phosphates, Colloids Surf. A: Physicochem. Eng. Aspects 247 (2004) 85-90.

[19] A.K. Dennis, C. Michel, N. Ganesan, Effect of thermal treatment on interfacia properties of $\beta$-lactoglobulin, J. Colloid Interface Sci. 285 (2005) 100-109.

[20] M.A. Rodriguez-Valverde, M.A. Cabrerizo-Vilchez, A. Paez-Duenas, R. HidalgoAlvarez, Stability of highly charged particles: bitumen-in-water dispersions, Colloids Surf. A: Physicochem. Eng. Aspects 222 (2003) 233-251.

[21] J.I. Acedo-Carrillo, A. Rosas-Durazo, R. Herrera-Urbina, M. Rinaudo, F.M. Goycoolea, M.A. Valdez, Zeta potential and drop growth of oil in water emulsions stabilized with mesquite gum, Carbohydr. Polym. 65 (2006) 327-336.

[22] A. Lozsan, M. Garcia-Sucre, G. Urbina-Villalba, Theoretical estimation of stability ratios for hexadecane-in-water $(\mathrm{H} / \mathrm{W})$ emulsions stabilized with nonylphenol ethoxylated surfactants, J. Colloid Interface Sci. 299 (2006) 366-377.

[23] N. Lazaridis, A.H. Alexopoulos, E.G. Chatzi, C. Kiparissides, Steric stabilization in emulsion polymerization using oligomeric nonionic surfactants, Chem. Eng. Sci. 54 (1999) 3251-3261.

[24] A. Hanke, E. Eisenriegler, S. Dietrich, Polymer depletion effects near mesoscopic particles, Phys. Rev. E 59 (1999) 6853-6878.

[25] X. Yang, W. Lu, Advances in stabilization and destabilization of water-in-crude oil emulsion, Oilfield Chem. 15 (1998) 87-96.

[26] P.C. Schorling, D.G. Kessel, I. Rahimian, Influence of the crude oil resin/asphaltene ratio on the stability of oil/water emulsions, Colloids Surf. A: Physicochem. Eng. Aspects 152 (1999) 95-102.

[27] M. Li, M. Lin, Z. Wu, A.C. Alfred, The influence of $\mathrm{NaOH}$ on the stability of paraffinic crude oil emulsion, Fuel 84 (2004) 183-187.

[28] C. Ci, J. Xu, J. Gong, Q. Yan, The mechanism and control methods of nonmagnetics polluted coal processing by flotation, J. Anhui U. S. T. 24 (2005) 74-77.

[29] C.B. Mark, R.H. Brian, P. Russell, A.K. Brad, C.S. Roger St, Analysis of mineral surface chemistry in flotation separation using imaging XPS, Miner. Eng. 20 (2007) 152-162. 